\title{
CONSTRUCTION OF POLYNOMIALS, ORTHOGONAL ON A DISCRETE SET OF POINTS
}

\author{
Inna Nikolova \\ Tzarigradsko shose 125, Block 2 \\ Institute of Information and \\ Communication Technologies \\ Bulgarian Academy of Sciences \\ Sofia 1113, BULGARIA
}

\begin{abstract}
In this paper we construct the set of Charlier polynomials, Krawtchouk polynomials and Hahn polynomials starting from their weight functions. We also compute their norms and moments.
\end{abstract}

AMS Subject Classification: 33C05, 33C45, 33C47, 42C05

Key Words: special functions; orthogonal polynomials

\section{Introduction}

In this paper we follow the approach of constructing the polynomials, orthogonal w.r.t. the weight functions, supported on a discrete set of points. Starting with the weight function we suppose that the polynomials are written in the form

$$
p_{n}(x)=\sum_{k=0}^{n} a_{n, k}(-x)_{k} .
$$

In the above equation $(-x)_{k}=(-x) \cdot(-x+1) \ldots(-x+k-1)$ is used. Write down the corresponding expression for the moments of the polynomials. From the orthogonality condition we guess the form of the coefficients $a_{n, k}$ and thus

Received: September 1, 2021

(C) 2021 Academic Publications 
construct the polynomial sequence. We consider only weights supported on a discrete set of points.

Definition 1. In a discrete setting a weight function is a positive function, defined on a discrete set $A$, which is typically finite or countable.

We consider the vector space of functions with integrable square over the field of real numbers. In the discrete setting the integral reducesees to finite of infinite sum.

Definition 2. Inner product is a way to multiply vectors together with the result of this multiplication being a scalar. It satisfies the following 3 properties: 1. Linearity w.r.t. the first argument:

$$
\begin{gathered}
\langle u+v, w\rangle=\langle u, v\rangle+\langle v, w\rangle, \\
\langle\alpha v, w\rangle=\alpha\langle v, w\rangle .
\end{gathered}
$$

2. Symmetry

$$
\langle v, w\rangle=\langle w, v\rangle .
$$

3. Positive definiteness

$$
\langle v, v\rangle \geq 0,\langle v, v\rangle=0 \text { iff } v=0 .
$$

Given a weight function, we define an inner product by

$$
\langle u, v\rangle=\sum_{l=0}^{t} u(l) v(l) w(l),
$$

where $t$ may be finite or infinite.

Definition 3. The norm of the vector in the considered space is given by

$$
\kappa=\sqrt{\langle v, v\rangle} .
$$

Definition 4. The $j$-th moment of the polynomial $p_{n}(x)$ is the inner product

$$
M_{j}\left(p_{n}(x)\right)=\left\langle x^{j}, p_{n}(x)\right\rangle .
$$




\section{Charlier polynomials}

Theorem 5. The Charlier polynomials are given by

$$
C_{n}(x)=\sum_{k=0}^{n} \frac{(-n)_{k}(-x)_{k}}{k !(-a)^{k}}
$$

They are orthogonal w.r.t. the weight function $a^{x} / x !$. The moments of Charlier polynomials are equal to

$$
\left\langle x^{j}, C_{n}(x)\right\rangle=a^{j}(-j)_{n} \frac{1}{a^{n}} e^{a} .
$$

The square of the norm of Charlier polynomials is

$$
\kappa_{n}^{2}=\frac{n ! e^{a}}{a^{n}}
$$

Proof. Suppose that the polynomials are represented by

$$
C_{n}(x)=\sum_{k=0}^{n} a_{n, k}(-x)_{k}
$$

We are looking for coefficients $a_{n, k}$ such that the moments

$$
M_{j}=\left\langle x^{j}, C_{n}(x)\right\rangle=0 \text { for } j<n .
$$

Fix $n$. Let us compute the inner product:

$$
\begin{aligned}
& M_{j}=\left\langle(-x)_{j}, C_{n}(x)\right\rangle \\
= & \sum_{l=0}^{\infty}(-l)_{j} \sum_{k=0}^{n} a_{n, k}(-l)_{k} \frac{a^{l}}{l !} \\
= & \sum_{l=0}^{\infty} \sum_{k=0}^{n} a_{n, k}(-l)_{j}(-l)_{k} \frac{a^{l}}{l !} .
\end{aligned}
$$

But $(-l)_{j}=0$ for $l \leq j$. Therefore we can reduce the limits of summation as

$$
M_{j}=\sum_{l=j}^{\infty} \sum_{k=0}^{n} a_{n, k}(-l)_{j}(-l)_{k} \frac{(-1)^{j} l ! a^{l}}{l !(l-j) !}
$$




$$
\begin{aligned}
= & (-1)^{j} \sum_{l=j}^{\infty} \sum_{k=0}^{n} a_{n, k}(-l)_{k} \frac{a^{l}}{(l-j) !} \\
= & (-1)^{j} \sum_{l=0}^{\infty} \sum_{k=0}^{n} a_{n, k}(-l-j)_{k} \frac{a^{l+j}}{l !} \\
= & (-1)^{j} \sum_{l=0}^{\infty} a_{n, 0} \frac{a^{l+j}}{l !} \\
& +(-1)^{j} \sum_{l=0}^{\infty} \sum_{k=1}^{n} a_{n, k}(-l-j)_{k} \frac{a^{l+j}}{l !} .
\end{aligned}
$$

But

$$
\begin{aligned}
(-l-j)_{1} & =(-l)_{1}+(-j)_{1} \\
(-l-j)_{k} & =(-l)_{k}+\sum_{s=1}^{k-1}\left(\begin{array}{l}
k \\
s
\end{array}\right)(-j)_{s}(-l)_{k-s}+(-j)_{k}, \quad k \geq 2 .
\end{aligned}
$$

The above representation can be proven by induction. After substitution we get:

$$
\begin{aligned}
& \quad\left\langle(-x)_{j}, C_{n}(x)\right\rangle \\
& =(-1)^{j} \sum_{l=0}^{\infty} a_{n, 0} \frac{a^{l+j}}{l !} \\
& +(-1)^{j} \sum_{l=0}^{\infty} a_{n, 1} a^{l}(-l)_{1}+(-1)^{j} a^{j}(-j)_{1} \sum_{l=0}^{\infty} \frac{a^{l}(-l)_{1}}{l !} \\
& +(-1)^{j} a^{j} \sum_{l=0}^{\infty} \sum_{k=2}^{n} a_{n, k} a^{l} \frac{(-l-j)_{k}}{l !} \\
& =(-1)^{j} \sum_{l=0}^{\infty} a_{n, 0} \frac{a^{l+j}}{l !} \\
& +(-1)^{j} a^{j} \sum_{l=0}^{\infty} \sum_{k=1}^{n-1} a_{n, k} a^{l}\left[(-l)_{k}\right. \\
& +\sum_{k-1}\left(\begin{array}{l}
k \\
s
\end{array}\right)^{\left.(-j)_{s}(-l)_{k-s}+(-j)_{k}\right] .}
\end{aligned}
$$

The triple sum above is absolutely convergent as a combination of absolutely convergent series and double finite sum. Therefore, we can change the order of 
summation and can express the triple sum as a table of infinite sums. Indeed, if we denote the $l-t h$ element of the above infinite sum by $b_{l}$ we obtain the following inequality:

$$
\begin{aligned}
\left|b_{l}\right| & \leq \sum_{k=0}^{n} \max _{0 \leq k \leq n}\left|a_{n, k}\right| \sum_{s=0}^{n}\left|\left(\begin{array}{c}
k \\
s
\end{array}\right) j^{s} l^{k-s}\right| \frac{a^{l}}{l !} \\
& \leq(n+1) \times \max _{0 \leq k \leq n}\left|a_{n, k}\right| 2^{n} j^{n} l^{n} \frac{l^{n} a^{l}}{l !} \\
& =C(n) \frac{l^{n} a^{l}}{l !} .
\end{aligned}
$$

Therefore the change of summation is legal,

$$
\begin{aligned}
& M_{j}=(-1)^{j} a^{j} a_{n, 0} \sum_{l=0}^{\infty} \frac{a^{l}}{l !} \\
& +(-1)^{j} a^{j} a_{n, 1} \sum_{l=0}^{\infty} \frac{(-l)_{1}}{l !} a^{l}+(-1)^{j} a^{j} a_{n, 1}(-j)_{1} \sum_{l=0}^{\infty} \frac{a^{l}}{l !} \\
& +(-1)^{j} a^{j} a_{n, k} \sum_{l=0}^{\infty} \frac{(-l)_{k}}{l !} a^{l} \\
& +\quad(-1)^{j} a^{j} a_{n, k}\left(\begin{array}{l}
k \\
1
\end{array}\right)(-j)_{1} \sum_{l=0}^{\infty} \frac{(-l)_{k-1}}{l !} a^{l} \\
& +\quad(-1)^{j}(-j)_{2} a^{j} a_{n, k}\left(\begin{array}{c}
k \\
2
\end{array}\right) \sum_{l=0}^{\infty} \frac{(-l)_{k-2} a^{l}}{l !}+\ldots \\
& +\quad(-1)^{j} a^{j}(-j)_{k} a_{n, k}\left(\begin{array}{l}
k \\
k
\end{array}\right) \sum_{l=0}^{\infty} \frac{a^{l}}{l !} \\
& +(-1)^{j} a^{j} a_{n, n} \sum_{l=0}^{\infty} \frac{(-l)_{n} a^{l}}{l !} \\
& +(-1)^{j} a^{j} a_{n, n}\left(\begin{array}{c}
n \\
1
\end{array}\right)(-j)_{1} \sum_{l=0}^{\infty} \frac{(-l)_{n-1} a^{l}}{l !} \\
& +(-1)^{j} a^{j} a_{n, n}(-j)_{2}\left(\begin{array}{l}
n \\
2
\end{array}\right) \sum_{l=0}^{\infty} \frac{(-l)_{n-2} a^{l}}{l !}
\end{aligned}
$$




$$
\begin{gathered}
+(-1)^{j}(-j)_{k} a^{j} a_{n, n}\left(\begin{array}{l}
n \\
k
\end{array}\right) \sum_{l=0}^{\infty} \frac{(-l)_{n-k} a^{l}}{l !}+\ldots \\
+(-1)^{j} a_{n, n}(-j)_{n} a^{j}\left(\begin{array}{l}
n \\
n
\end{array}\right) \sum_{l=0}^{\infty} \frac{a^{l}}{l !} .
\end{gathered}
$$

Choose

$$
\begin{aligned}
& a_{n, k}=\frac{(-n)_{k}}{k !(-a)^{k}}, \quad a>0 . \\
& M_{j}=(-1)^{j} a^{j} e^{a} \\
& +(-1)^{j} a^{j} \frac{(-n)_{1}}{(-a)^{1} 1 !} \sum_{l=1}^{\infty} \frac{(-1) a^{l}}{(l-1) !}+(-1)^{j} a^{j} \frac{(-n)_{1}}{(-a)^{1} 1 !}(-j)_{1} e^{a} \\
& +(-1)^{j} a^{j} \frac{(-n)_{2}}{(-a)^{2} 2 !} \sum_{l=2}^{\infty} \frac{(-1)^{2} l ! a^{l}}{l !(l-2) !}+ \\
& +(-1)^{j} a^{j} \frac{(-n)_{2}}{(-a)^{2} 2 !}\left(\begin{array}{l}
2 \\
1
\end{array}\right)(-j)_{1} \sum_{l=1}^{\infty} \frac{(-1) l !}{(l-1) ! l !} a^{l} \\
& +(-1)^{j}(-j)_{2} a^{j} \frac{(-n)_{2}}{(-a)^{2} 2 !}\left(\begin{array}{l}
2 \\
2
\end{array}\right) \sum_{l=0}^{\infty} \frac{a^{l}}{l !} \\
& +(-1)^{j} a^{j} \frac{(-n)_{3}}{(-a)^{3} 3 !} \sum_{l=3}^{\infty} \frac{(-1)^{3} a^{l} l !}{l !(l-3) !} \\
& +(-1)^{j} a^{j} \frac{(-n)_{3}}{(-a)^{3} 3 !}\left(\begin{array}{l}
3 \\
1
\end{array}\right)(-j)_{1} \sum_{l=2}^{\infty} \frac{(-1)^{2} l !}{(l-2) !} a^{l} \\
& +\quad(-1)^{j}(-j)_{2} a^{j} \frac{(-n)_{3}}{(-a)^{3} 3 !}\left(\begin{array}{l}
3 \\
2
\end{array}\right) \sum_{l=1}^{\infty} \frac{(-1) l !}{l !(l-1) !} a^{l} \\
& +(-1)^{j}(-j)_{3} a^{j} \frac{(-n)_{3}}{(-a)^{3} 3 !}\left(\begin{array}{l}
3 \\
3
\end{array}\right) e^{a} \\
& +(-1)^{j} a^{j} \frac{(-n)_{k}}{(-a)^{k} k !} \sum_{l=k}^{\infty} \frac{(-1)^{k} l ! a^{l}}{l !(l-k) !} \\
& +(-1)^{j} a^{j} \frac{(-n)_{k}}{(-a)^{k} k !}\left(\begin{array}{l}
k \\
1
\end{array}\right)(-j)_{1} \sum_{l=k-1}^{\infty} \frac{(-1)^{k-1} l ! a^{l}}{(l-k+1) ! l !}
\end{aligned}
$$




$$
\begin{aligned}
& +\quad(-1)^{j} a^{j}(-j)_{2} \frac{(-n)_{k}}{(-a)^{k} k !}\left(\begin{array}{l}
k \\
2
\end{array}\right) \sum_{l=k-2}^{\infty} \frac{(-1)^{k-2} l ! a^{l}}{l !(l-k+2) !}+ \\
& \cdots+(-1)^{j}(-j)_{k} \frac{(-n)_{k}}{(-a)^{k} k !}\left(\begin{array}{l}
k \\
k
\end{array}\right) e^{a} \\
& +(-1)^{j} a^{j} \frac{(-n)_{n}}{(-a)^{n} n !} \sum_{l=n}^{\infty} \frac{(-1)^{n} a^{l} l !}{l !(l-n) !} \\
& +(-1)^{j} a^{j} \frac{(-n)_{n}}{(-a)^{n} n !}\left(\begin{array}{c}
n \\
1
\end{array}\right)(-j)_{1} \sum_{l=n-1}^{\infty} \frac{(-1)^{n-1} l ! a^{l}}{(l-n+1) ! l !} \\
& +\quad(-1)^{j} a^{j}(-j)_{2} \frac{(-n)_{n}}{(-a)^{n} n !}\left(\begin{array}{l}
n \\
2
\end{array}\right) \sum_{l=n-2}^{\infty} \frac{(-1)^{n-2} a^{l} l !}{l !(l-n+2) !} \\
& +\cdots+(-1)^{j} a^{j}(-j)_{n} \frac{1}{n !} \frac{(-n)_{n}}{(-a)^{n} n !} e^{a} \\
& =(-1)^{j} a^{j} e^{a} \\
& +(-1)^{j} a^{j} \frac{(-n)_{1}}{(-a)^{1} 1 !}(-a) e^{a}+(-1)^{j} a^{j} \frac{(-n)_{1}}{(-a)^{1} 1 !}(-j)_{1} e^{a} \\
& =(-1)^{j} a^{j} \frac{(-n)_{2}}{(-a)^{2} 2 !}(-a)^{2} e^{a} \\
& +(-1)^{j} a^{j} \frac{(-n)_{2}}{(-a)^{2} 2 !}\left(\begin{array}{l}
2 \\
1
\end{array}\right)(-j)_{1}(-a) e^{a} \\
& +\quad(-1)^{j}(-j)_{2} a^{j}\left(\begin{array}{l}
2 \\
2
\end{array}\right) \frac{(-n)_{2}}{(-a)^{2} 2 !} e^{a} \\
& +(-1)^{j} a^{j} \frac{(-n)_{k}}{(-a)^{k} k !}(-a)^{k} e^{a} \\
& +(-1)^{j} a^{j} \frac{(-n)_{k}}{(-a)^{k} k !}\left(\begin{array}{c}
k \\
1
\end{array}\right)(-j)_{1}(-a)^{k-1} e^{a} \\
& +\quad(-1)^{j}(-j)_{2} a^{j}\left(\begin{array}{l}
k \\
2
\end{array}\right) \frac{(-n)_{k}}{(-a)^{k} k !}(-a)^{k-2} e^{a}+ \\
& +(-1)^{j}(-j)_{k} a^{j}\left(\begin{array}{l}
k \\
k
\end{array}\right) \frac{(-n)_{k}}{(-a)^{k} k !} e^{a}
\end{aligned}
$$

$\vdots$ 


$$
\begin{aligned}
& +(-1)^{j} a^{j} \frac{(-n)_{n}}{(-a)_{n} n !}(-a)^{n} e^{a} \\
& +\quad(-1)^{j} a^{j} \frac{(-n)_{n}}{(-a)^{n} n !}\left(\begin{array}{c}
n \\
1
\end{array}\right)(-j)_{1}(-a)^{n-1} e^{a} \\
& +\quad(-1)^{j}(-j)_{2} a^{j}\left(\begin{array}{c}
n \\
2
\end{array}\right) \frac{(-n)_{n}}{(-a)^{n} n !}(-a)^{n-2} e^{a}+\ldots \\
& +(-1)^{j}(-j)_{n} a^{j}\left(\begin{array}{c}
n \\
n
\end{array}\right) \frac{(-n)_{n}}{(-a)^{n} n !} e^{a} .
\end{aligned}
$$

After substituting

$$
\left(\begin{array}{l}
k \\
s
\end{array}\right)=\frac{k !}{s !(k-s) !},
$$

we receive that in the above table the first column equals to

$$
(-1)^{j} a^{j} e^{a} \sum_{k=0}^{n} \frac{(-n)_{k}}{k !}=0 .
$$

The sum of elements in the second column is equal to

$$
\begin{aligned}
& (-1)^{j} a^{j} e^{a}(-j)_{1} \sum_{k=1}^{n} \frac{(-n)_{k}}{(-a)(k-1) !} \\
= & (-1)^{j} a^{j} e^{a} \frac{-n}{-a} \sum_{k=0}^{n-1} \frac{(-n+1)_{k}}{k !}=0 .
\end{aligned}
$$

The sum of elements in the column 3 is equal to

$$
(-1)^{j}(-j)_{2} a^{j} \frac{(-n)_{2}}{(-a)^{2} 2 !} \sum_{k=0}^{n-2} \frac{(-n+2)_{k}}{k !}=0 .
$$

And so on, until we reach the last column which consist of one element, namely

$$
(-1)^{j} a^{j}(-j)_{n} \frac{(-n)_{n}}{(-a)^{n} n !} e^{a}
$$

Therefore

$$
M_{j}=\left\langle(-x)_{j}, C_{n}(x)\right\rangle=(-1)^{j}\left\langle x^{j}, C_{n}(x)\right\rangle .
$$


When $j=n$ we get

$$
\left\langle x^{n}, C_{n}(x)\right\rangle=(-1)^{n} n ! e^{a} .
$$

But

$$
\gamma_{n}=\frac{(-n)_{n}(-1)^{n}}{n !(-a)^{n}}=\frac{1}{(-a)^{n}}
$$

Therefore

$$
\left\langle\gamma_{n} x^{n}, C_{n}(x)=\frac{n ! e^{a}}{a^{n}} .\right.
$$

So the square of the norm is equal to:

$$
\kappa_{n}^{2}=\frac{(n !) e^{a}}{a^{n}} .
$$

The expression for the Charlier polynomials together with their weight function and the square of the norm are given in [1] without proof. The moments are not considered there. In the work [2] different definition of the moments is used, and because of that the explicit expression for the moments is not found. In the work [3] some facts about Charlier polynomials are given but the expression for the moments is also missing.

\section{Krawtchouk polynomials}

Theorem 6. The Krawtchouk polynomials are defined by

$$
K_{n}(x, p, N)=K_{n}(x)=\sum_{k=0}^{n} \frac{(-n)_{k}(-x)_{k}}{k !(-N)_{k}(-p)^{k}}
$$

They are orthogonal w.r.t. the weight function $\left(\begin{array}{c}N \\ x\end{array}\right) p^{x}(1-p)^{N-x}$. The moments of the Krawtchouk polynomials are equal to

$$
\left\langle x^{j}, K_{n}(x, p, N)\right\rangle=\frac{(-N)_{j}(1-p)^{j}(-j)_{n}}{(-N)_{n}} .
$$

The square of the norm of the Krawtchouk polynomials is

$$
\kappa_{n}^{2}=\frac{(-n)_{n}}{(-N)_{n}} \frac{(1-p)^{n}}{p^{n}}
$$


Proof. The weight function of the Krawtchouk polynomials is given by

$$
\begin{aligned}
w(l) & =\left(\begin{array}{c}
N \\
l
\end{array}\right) p^{l}(1-p)^{N-l} \\
& =\frac{N ! p^{l}(1-p)^{N-l}}{l !(N-l) !}=\frac{(-N)_{l}(-p)^{l}(1-p)^{N-l}}{l !} .
\end{aligned}
$$

Suppose that the polynomials orthogonal w.r.t. this weight function are written in the following way:

$$
K_{n}(x)=\sum_{k=0}^{n} a_{n, k}(-x)_{k} .
$$

We are looking for $a_{n, k}$ such that

$$
\left\langle(-N+x)_{j}, K_{n}(x)\right\rangle=0 \text { for } j<n .
$$

But

$$
\begin{aligned}
& \left\langle(-N+x)_{j}, K_{n}(x)\right\rangle \\
= & \sum_{l=0}^{N} \frac{(-N+l)_{j}(-N)_{l}(-p)^{l}(1-p)^{N-l}}{l !} \sum_{k=0}^{n} a_{n, k}(-l)_{k} \\
= & \sum_{k=0}^{n} a_{n, k} \sum_{l=k}^{N} \frac{(-N)_{l+j}(-p)^{l}(1-p)^{N-l}(-l)_{k}}{l !} .
\end{aligned}
$$

In the last equation we used the fact that $(-l)_{k}=0$ for $l<k$. For $l \geq k$ the following holds

$$
(-l)_{k}=\frac{(-1)^{k} l !}{(l-k) !}
$$

Then,

$$
\begin{aligned}
& \left\langle(-N+x)_{j}, K_{n}(x)\right\rangle \\
= & \sum_{k=0}^{n} a_{n, k} \sum_{l=k}^{N} \frac{(-N)_{l+j}(-p)^{l}(1-p)^{N-l}(-1)^{k} l !}{l !(l-k) !} \\
= & \sum_{k=0}^{n} a_{n, k} \sum_{l=0}^{N-k} \frac{(-N)_{l+j+k}(-p)^{l+k}(1-p)^{N-k-l}(-1)^{k}}{l !} .
\end{aligned}
$$

We are going to use the following relations

$$
(-N)_{l+j+k}=(-N)_{j}(-N+j)_{k}(-N+j+k)_{l},
$$




$$
(-p)^{l+k}(1-p)^{N-l-k}=(1-p)^{N}\left(-\frac{p}{1-p}\right)^{l}\left(-\frac{p}{1-p}\right)^{k}
$$

Then (8) can be written as

$$
\begin{aligned}
& =(-N)_{j}(1-p)^{N} \sum_{k=0}^{n} a_{n, k}(-1)^{k} \\
& \times\left(-\frac{p}{1-p}\right)^{k}(-N+j)_{k} \\
& \times \sum_{l=0}^{N-k} \frac{(-N+j+k)_{l}}{l !}\left(-\frac{p}{1-p}\right)^{l} \\
& =(-N)_{j}(1-p)^{N} \sum_{k=0}^{n} a_{n, k}(-1)^{k}(-N+j)_{k} \\
& \times\left(-\frac{p}{1-p}\right)^{k}\left(1+\frac{p}{1-p}\right)^{N-j-k} \\
& =(-N)_{j}(1-p)^{N} \sum_{k=0}^{n} a_{n, k} \\
& \times \frac{p^{k}(-N+j)_{k}}{(1-p)^{k}} \\
& \times\left(1+\frac{p}{1-p}\right)^{N-j-k} \\
& =(-N)_{j}(1-p)^{N} \sum_{k=0}^{n} a_{n, k} \\
& \times \frac{p^{k}(-N+j)_{k}}{(1-p)^{N-j}} \\
& =(-N)_{j}(1-p)^{j} \sum_{k=0}^{n} a_{n, k} p^{k}(-N+j)_{k} \text {. }
\end{aligned}
$$

Here we used the binomial theorem. Namely,

$$
(1-z)^{-a}=\sum_{k=0}^{\infty} \frac{(a)_{k} z^{k}}{k !}
$$

From here it follows that 


$$
\left\langle(-N+x)_{j}, K_{n}(x)\right\rangle=(-N)_{j}(1-p)^{j} \sum_{k=0}^{n} a_{n, k}(-N+j)_{k} p^{k} .
$$

The following choice leads to the desired result:

$$
a_{n, k}=\frac{(-n)_{k}}{k !(-N)_{k}} \frac{1}{p^{k}} .
$$

Namely:

$$
\begin{aligned}
\left\langle(-N+x)_{j}, K_{n}(x)\right\rangle & =(-N)_{j}(1-p)^{j} \sum_{k=0}^{n} \frac{(-n)_{k}(-N+j)_{k}}{k !(-N)_{k}} \\
& =(-N)_{j}(1-p)^{j} \frac{(-N+N-j)_{n}}{(-N)_{n}} \\
& =\frac{(-N)_{j}(1-p)^{j}(-j)_{n}}{(-N)_{n}} .
\end{aligned}
$$

The last expression is equal to zero for $j<n$. For $j=n$ it takes the form:

$$
\left\langle(-N+x)_{n}, K_{n}(x)\right\rangle=(-n)_{n}(1-p)^{n} .
$$

From the above statement it follows that

$$
\left\langle(-N+x)_{n}, K_{n}(x)\right\rangle=\left\langle(x)^{n}, K_{n}(x)\right\rangle .
$$

Therefore the polynomials, orthogonal w.r.t. this weight, take the following form

$$
K_{n}(x)=\sum_{k=0}^{n} \frac{(-n)_{k}(-x)_{k}}{k !(-N)) k} \frac{1}{p^{k}} .
$$

Since the leading coefficient $\gamma_{n}$ is equal to

$$
\gamma_{n}=\frac{(-n)_{n}(-1)^{n}}{n !(-N)_{n}} \frac{1}{p^{n}},
$$

the expression for the norm becomes:

$$
\left\langle\gamma_{n}(-N+x)_{n}, K_{n}(x)\right\rangle=\left\|K_{n}(x)\right\|^{2}=\frac{(-n)_{n}}{(-N)_{n}} \frac{(1-p)^{n}}{p^{n}} .
$$

In the work [4] a different definition for the moments is used and therefore they are not obtained in the sense we consider here. The expressions for the Krawtchouk polynomials, their weight function and the norm of the polynomials are given in [1] without proof. 


\section{Hahn polynomials}

Theorem 7. The weight function of the Hahn polynomials is given by

$$
w(x)=\frac{(\alpha+1)_{x}(\beta+1)_{N-x}}{x !(N-x) !},
$$

where $x=0,1, \ldots, N$. The moments of the Hahn polynomials are equal to

$$
\begin{aligned}
M_{j}\left(Q_{n}(x)\right) & =\left\langle x^{j}, Q_{n}(x)\right\rangle=\left\langle(\alpha+x+1)_{j}, Q_{n}(x)\right\rangle \\
& =\frac{(\alpha+1)_{j}(\alpha+\beta+j+2)_{N}(\beta+1)_{n}(-j)_{n}}{(\alpha+\beta+j+2)_{n} N !(\alpha+1)_{n}} .
\end{aligned}
$$

And the square of the norm of the Hahn polynomials is equal to

$$
\left\langle Q_{n}(x), Q_{n}(x)\right\rangle=\frac{(\beta+1)_{n}(\alpha+\beta+n+1)_{N+1}(-n)_{n}}{(\alpha+1)_{n}(\alpha+\beta+2 n+1)(-N)_{n}} .
$$

Proof. Here we are deriving the expression for the moments. We are looking for polynomials of the form

$$
Q_{n}(x)=\sum_{k=0}^{n} a_{n, k}(-x)_{k}
$$

such that for every $n=0,1, \ldots, N$ to hold:

$$
\left\langle(\alpha+x+1)_{j}, Q_{n}(x)\right\rangle=0 \quad \text { for } j<n .
$$

From the following proof it is obvious that this expression is equal to the moments

$$
\begin{gathered}
\left\langle x^{j}, Q_{n}(x)\right\rangle . \\
\left\langle(\alpha+x+1)_{j}, Q_{n}(x)\right\rangle \\
=\sum_{l=0}^{N} \frac{(\alpha+l+1)_{j} Q_{n}(l)(\alpha+1)_{l}(\beta+1)_{N-l}}{l !(N-l) !} \\
=\sum_{l=0}^{N} \frac{(\alpha+1)_{l+j}(\beta+1)_{N-l} Q_{n}(l)}{l !(N-l) !} \\
=\sum_{l=0}^{N} \frac{(\alpha+1)_{l+j}(\beta+1)_{N-l}}{l !(N-l) !} \sum_{k=0}^{n} a_{n, k}(-l)_{k} .
\end{gathered}
$$


We are going to change the order of summation. Since we are dealing with finite sums this operation is legal. Moreover, we are going to use the fact that $(-l)_{k}=0$ for $l<k$. That is, the above finite sum has non zeros entries only if $l \geq k$. Therefore the following is true:

$$
\begin{aligned}
& \left\langle(\alpha+x+1)_{j}, Q_{n}(x)\right\rangle \\
= & \sum_{k=0}^{n} \sum_{l=k}^{N} \frac{a_{n, k}(\alpha+1)_{l+j}(\beta+1)_{N-l}(-l)_{k}}{l !(N-l) !} .
\end{aligned}
$$

When $l \geq k$ the following holds:

$$
(-l)_{k}=\frac{(-1)^{k} l !}{(l-k) !} .
$$

After substituting this expression in (14) we get that

$$
\begin{aligned}
& \left\langle(\alpha+x+1)_{j}, Q_{n}(x)\right\rangle \\
= & \sum_{k=0}^{n} \sum_{l=k}^{N} \frac{a_{n, k}(\alpha+1)_{l+j}(\beta+1)_{N-l}(-1)^{k} l !}{(N-l) !(l-k) ! l !} \\
= & \sum_{k=0}^{n} \sum_{l=0}^{N-k} \frac{a_{n, k}(\alpha+1)_{l+k+j}(\beta+1)_{N-l-k}(-1)^{k}}{(N-l-k) ! l !} .
\end{aligned}
$$

We are going to use the following relations:

$$
\begin{aligned}
(\alpha+1)_{l+k+j} & =(\alpha+1)_{j}(\alpha+j+1)_{k}(\alpha+j+k+1)_{l}, \\
(N-l-k) ! & =\frac{1.2 \ldots(N-l-k)(N-l-k+1) \ldots N}{(N-l-k+1) \ldots N} \\
& =\frac{N !(-1)^{l+k}}{(-N)_{l+k}}=\frac{N !(-1)^{l+k}}{(-N)_{k}(-N+k)_{l}},
\end{aligned}
$$

and

$$
\begin{aligned}
(\beta+1)_{N-l-k} & =\frac{(\beta+1) \ldots(\beta+N-l-k+1) \ldots(\beta+N)}{(\beta+N-l-k+1) \ldots(\beta+N)} \\
& =\frac{(\beta+1)_{N}(-1)^{l+k}}{(-\beta-N)_{l+k}} \\
& =\frac{(\beta+1)_{N}(-1)^{l+k}}{(-\beta-N)_{k}(-\beta-N+k)_{l}},
\end{aligned}
$$


to obtain that

$$
\begin{aligned}
& \left\langle(\alpha+x+1)_{j}, Q_{n}(x)\right\rangle \\
= & \frac{(\alpha+1)_{j}(\beta+1)_{N}}{N !} \sum_{k=0}^{n} \frac{a_{n, k}(-N)_{k}(-1)^{k}(\alpha+j+1)_{k}}{(-\beta-N)_{k}} \times \\
& \sum_{l=0}^{N-k} \frac{(\alpha+j+k+1)_{l}(-N+k)_{l}}{l !(-\beta-N+k)_{l}} .
\end{aligned}
$$

From here, using the Chu-Vandermonde theorem:

$$
\sum_{k=0}^{n} \frac{(-n)_{k}(a)_{k}}{(b)_{k}}=\frac{(b-a)_{n}}{(b)_{n}}
$$

we get that

$$
\begin{aligned}
& \left\langle(\alpha+x+1)_{j}, Q_{n}(x)\right\rangle \\
& =\frac{(\alpha+1)_{j}(\beta+1)_{N}}{N !} \\
& \times \sum_{k=0}^{n} \frac{a_{n, k}(-1)^{k}(\alpha+j+1)_{k}(-N)_{k}(-\beta-N-\alpha-j-1)_{N-k}}{(-\beta-N)_{k}(-\beta-N+k)_{N-k}}, \\
& (-\beta-N-\alpha-j-1)_{N-k}=\frac{(-\beta-N-\alpha-j-1)_{N}(-1)^{k}}{(1+\alpha+\beta+j+1)_{k}} \\
& =\frac{(-1)^{N-k}(\alpha+\beta+j+2)_{N}}{(\alpha+\beta+j+2)_{k}} \text {, } \\
& (-\beta-N+k)_{N-k} \\
& =(-\beta-N+k)(-\beta-N+k+1) \ldots \\
& \ldots(-\beta-N+k+N-k-1) \\
& =(-1)^{N-k}(\beta+1)_{N-k} \\
& =\frac{(-1)^{N}(\beta+1)_{N}}{(-\beta-N)_{k}} \text {. }
\end{aligned}
$$

Let

$$
a_{n, k}=\frac{(-n)_{k}(\alpha+\beta+n+1)_{k}}{k !(\alpha+1)_{k}(-N)_{k}}
$$


Then

$$
\begin{aligned}
& \left\langle(\alpha+x+1)_{j}, Q_{n}(x)\right\rangle \\
= & \frac{(\alpha+1)_{j}(\alpha+\beta+j+2)_{N}}{N !} \\
\times & \sum_{k=0}^{n} \frac{(-n)_{k}(\alpha+\beta+n+1)_{k}(\alpha+j+1)_{k}}{k !(\alpha+1)_{k}(\alpha+\beta+j+2)_{k}} .
\end{aligned}
$$

Moreover, the following holds:

$$
(-n)+(\alpha+\beta+n+1)+(\alpha+j+1)+1=(\alpha+1)+(\alpha+\beta+j+2)
$$

and therefore the Phaph-Saaltchutz theorem is applicable,

$$
\sum_{k=0}^{n} \frac{(-n)_{k}(a)_{k}(b)_{k}}{(c)_{k}(d)_{k}}=\frac{(c-a)_{n}(c-b)_{n}}{(c)_{n}(c-a-b)_{n}} i f(-n)+a+b+1=c+d .
$$

From here we get that

$$
\begin{aligned}
& \left\langle(\alpha+x+1)_{j}, Q_{n}(x)\right\rangle \\
& \frac{(\alpha+1)_{j}(\alpha+\beta+j+2)_{N}(j-n+1)_{n}(\beta+1)_{n}}{N !(\alpha+\beta+j+2)_{n}(-\alpha-n)_{n}} .
\end{aligned}
$$

Moreover the following equations hold:

$$
\begin{aligned}
(j+1-n)_{n} & =(-1)^{n}(-j)_{n} \\
(-n-\alpha)_{n} & =(-1)^{n}(\alpha+1)_{n} .
\end{aligned}
$$

Therefore,

$$
\begin{aligned}
& \left\langle(\alpha+x+1)_{j}, Q_{n}(x)\right\rangle \\
& \frac{(\alpha+1)_{j}(\alpha+\beta+j+2)_{N}(\beta+1)_{n}(-j)_{n}}{(\alpha+\beta+j+2)_{n} N !(\alpha+1)_{n}} .
\end{aligned}
$$

But $(-j)_{n}$ is equal to zero for $j<n$. Therefore

$$
\left\langle(\alpha+x+1)_{j} Q_{n}(x)=\left\langle x^{j}, Q_{n}(x)\right\rangle .\right.
$$

Let $j=n$. Then

$$
\left\langle(\alpha+x+1)_{n}, Q_{n}(x)\right\rangle=\frac{(\beta+1)_{n}(-n)_{n}(\alpha+\beta+n+2)_{N}}{N !(\alpha+\beta+n+2)_{n}} .
$$


In order to compute the norm we use the fact that

$$
\begin{aligned}
\gamma_{n} & =\frac{(-n)_{n}(\alpha+\beta+n+1)_{n}(-1)^{n}}{n !(\alpha+1)_{n}(-N)_{n}} \\
& =\frac{(\alpha+\beta+n+1)_{n}}{(\alpha+1)_{n}(-N)_{n}},
\end{aligned}
$$

so we can obtain

$$
\begin{aligned}
\left\langle Q_{n}(x), Q_{n}(x)\right\rangle & =\gamma_{n}\left\langle(x+\alpha+1)_{n}, Q_{n}(x)\right\rangle \\
& =\frac{(-n)_{n}(\beta+1)_{n}(\alpha+\beta+n+2)_{N}(\alpha+\beta+n+1)_{n}}{N !(\alpha+\beta+n+2)_{n}(\alpha+1)_{n}(-N)_{n}} \\
& =\frac{(\beta+1)_{n}(\alpha+\beta+n+1)_{N+1}(-n)_{n}}{(\alpha+1)_{n}(\alpha+\beta+2 n+1)(-N)_{n}} .
\end{aligned}
$$

It is interesting to note that for $\alpha=\beta=0$ we obtain weight equal to unity for every point of the weight function. For these values of the parameters $\alpha$ and $\beta$ the Hahn polynomials look like

$$
Q_{n}^{0,0}(x)=\sum_{k=0}^{n} \frac{(-n)_{k}(n+1)_{k}(-x)_{k}}{k ! k !(-N)_{k}} .
$$

Since the weight function becomes equal to one for this case the Hahn polynomials in this case can be used in DSP. The definition of Hahn polynomials is given in [1].

\section{References}

[1] R. Koekoek, R. Swartouw, The Askey-scheme of hypergeometric orthogonal polynomials and its $q$-analogue, https://arxiv.org/pdf/math/9602214.pdf (1996).

[2] A.M. Abdul-Hadi, S.H. Abdulhussain and B.M. Mohammed, On the computational aspects of Charlier polynomials, Cogent Engineering, 7, No 1 (2020), Art. 1763553; DOI: 10.1080123311916.2020.1763553.

[3] G. Peccah, M.S. Taqqu, Some facts about Charlier polynomials, In: Wiener Chaos; Moments, Cumulants and Diagrams. Bocconi and Springer Ser., 1, Springer, Milano (2011); DOI: 10.1007/978-88-470-1679-8-10. 
[4] Pew-Thian Yap, Raveendran Paramesran, Seng-Huant Ong, Image analysis by Krawtchouk moment, IEEE Trans. on Image Processing 12, No 11 (Nov. 2003). 